\title{
Une politique de santé globale dans l'arène locale
}

La délivrance des antipaludéens à Bandjoun au Cameroun

\section{Albert Legrand Fosso}

\section{OpenEdition}

\section{Journals}

Édition électronique

URL : http://journals.openedition.org/anthropodev/375

DOI : 10.4000/anthropodev.375

ISSN : 2553-1719

Éditeur

APAD - Association pour l'anthropologie du changement social et du développement

Édition imprimée

Date de publication : 1 septembre 2015

Pagination : 161-195

ISBN : 979-10-93476-02-5

ISSN : 2276-2019

\section{Référence électronique}

Albert Legrand Fosso, "Une politique de santé globale dans l'arène locale », Anthropologie \&

développement [En ligne], 42-43 | 2015, mis en ligne le 01 décembre 2016, consulté le 19 avril 2019.

URL : http://journals.openedition.org/anthropodev/375 ; DOI : 10.4000/anthropodev.375

La revue Anthropologie \& développement est mise à disposition selon les termes de la Licence Creative Commons Attribution 4.0 International. 


\title{
Une politique de santé globale dans l'arène locale : la délivrance des antipaludéens à Bandjoun au Cameroun
}

\author{
Albert Legrand Fosso
}

La délivrance des combinaisons thérapeutiques à base d'artémisinine en traitement curatif du paludisme à Bandjoun (Ouest du Cameroun) dans le cadre de la "politique globale de la santé " est révélatrice de la déconnexion entre les enjeux globaux et locaux de la construction du marché de cet antipaludéen. À l'épreuve du local, les "prescriptions " globales de l'Organisation mondiale de la santé sont confrontées à des difficultés d'application. Cela tient à la complexité des circuits dans lesquels circulent les antipaludéens et à la pluralité des modes de délivrance locale des médicaments.

The delivery of the artemisinin-based combined therapy in the curative treatment of malaria in Bandjoun (West Cameroon) within the framework of the "global health policy" uncovers the disconnection between the global and local stakes in the construction of the market of this antimalarial drug. At the local level, the global "prescriptions" of the World Health Organization face implementation difficulties. This is due to the complexity of the networks in which antimalarial drugs move and the diversity of the local delivery system of the medicine.

\section{Introduction}

L'émergence à la fin des années 1990 du concept de "santé comme bien public mondial ${ }^{1}$ et l'avènement des "Programmes de santé » marquent

${ }^{1}$ Ce concept de "santé comme bien public mondial " est développé par A. Mills (2002). Notons que le concept de " bien public » tient de la théorie de l'économie publique standard (Samuelson, 1954) et désigne tout ce qui relève du domaine de la consommation (indistinc- 
le début d'une nouvelle ère dans le domaine des politiques de santé mondiales. Dans ce contexte, le paludisme devient une question de santé prioritaire qui mobilise la communauté internationale. Ce changement de paradigme qui s'opère avec le passage à la politique de "santé globale » basée sur la "gouvernance mondiale de la santé » est marqué par la création du Fonds mondial de lutte contre le sida, le paludisme et la tuberculose ainsi que la prise en compte de la lutte contre les grandes pandémies, dont le paludisme, par les Nations unies dans le cadre des Objectifs du millénaire pour le développement (OMD).

Ce regain d'intérêt pour la lutte contre le paludisme intervient au moment où, dans les pays endémiques d'Afrique subsaharienne, d'Asie du Sud-Est et d'Amérique du Sud, les résistances au traitement de cette maladie en monothérapie par la chloroquine sont sans cesse croissantes, et ce depuis la fin des années 1970. Ainsi, afin de remédier aux échecs thérapeutiques de la chloroquine, l'Organisation mondiale de la santé (OMS) " recommande" le passage aux combinaisons thérapeutiques à base d'artémisinine $(\mathrm{ACT})^{2}$ dans le cadre d'une "Stratégie mondiale » de lutte contre le paludisme. Toutefois, compte tenu du prix élevé des ACT par rapport à celui des anciens médicaments (de 0,90 à $2,40 \$$ pour les premiers contre 0,10 à $0,15 \$$ pour les seconds, en 2001$)^{3}$ et de la situation épidémiologique très inquiétante des pays affectés, I'OMS s'érige en " prescripteur global, en édictant d'un côté des recommandations de politique sanitaire en direction des pays endémiques et en organisant, de l'autre, une filière de production mondiale (d'ACT) " (Orsi et Zimmermann, $2014: 1)$.

tement de biens matériels et immatériels) qui n'est pas dans un rapport de rivalité ou d'exclusivité. Les biens publics sont non rivaux car leur consommation est durable, mieux "sustainable ". Leur utilité pour certains consommateurs ne diminue pas du fait de leur consommation par d'autres. Ils sont non exclusifs car leur usage est commun et ne peut être discriminant du fait notamment de leur coût économique.

${ }^{2}$ Dans cet article, nous utilisons le sigle anglais ACT (artemisinin-based combination therapy) plus connu que le français CTA.

${ }^{3}$ Il s'agit du " prix du coûtant » (prix de sortie d'usine ne dégageant aucun profit, ni perte) du Coartem, la première combinaison fixe d'ACT introduite en 1999. En 2001, le prix des ACT est vingt fois plus élevé que celui de la chloroquine (MSF, 2003). 
Les retours de terrain sur la question de la mise en œuvre de cette "gouvernance mondiale de la santé » en matière de lutte contre le paludisme en général, et celle toute particulière de l'introduction des ACT, révèlent que le passage du global au local s'est fait par une renégociation des directives internationales. Cela a été le cas lorsqu'il s'est agi de I'inscription des ACT dans les protocoles nationaux de traitement du paludisme au Cameroun (Fosso, 2013)4.

À la lumière des diverses perspectives des travaux sur la mise en œuvre du paradigme de la politique globale de la lutte contre le paludisme (Touré et Sanogo, 2014 ; Orsi et Zimmermann, 2014 ; Le Marcis et al., 2013), il apparaît que la "prescription " d'une politique ainsi que la disponibilité des moyens financiers n'assurent pas systématiquement l'application et l'efficacité sur le terrain des stratégies décidées en amont. Aussi les pratiques et les logiques des acteurs de la délivrance des biens et services de santé sont-elles à considérer, surtout lorsque ceux-ci s'illustrent par leur capacité à influencer tant la/le politique, que l'économie et le social. D'où l'intérêt que nous accordons à l'étude des pratiques et logiques des acteurs de la délivrance des ACT dans un espace local. Ainsi, comment opèrent ces acteurs locaux à Bandjoun ? Que révèle cette délivrance des ACT de l'application des directives globales de santé et de la connexion (ou déconnexion) entre les enjeux globaux et locaux ?

Cette étude se situe dans une perspective anthropologique de la configuration de délivrance des ACT et se fonde sur nos investigations de terrain menées dans le cadre de la recherche doctorale (2009-2014), dans le district sanitaire de Bandjoun situé à l'Ouest du Cameroun. D'un point de vue administratif, Bandjoun est le chef-lieu du département du Koung-Khi. Ce district sanitaire est composé d'un hôpital de district (hôpital de référence), de deux centres médicaux d'arrondissement et de quatorze centres

\footnotetext{
${ }^{4}$ Les difficultés de mise en œuvre des nouvelles recommandations en matière de traitement du paludisme se sont également révélées avec l'avènement de l' " evidence based medicine ». La principale nouveauté de cette approche est l'introduction des tests de diagnostic rapide (TDR) dont l'usage peine à entrer dans les habitudes des professionnels de santé, comme le montrent les travaux de F. Le Marcis et al. (2013) au Sénégal et L. Touré et Y.T. Sanogo (2014) au Mali.
} 
de santé intégrés $(\mathrm{CSI})^{5}$. L'analyse de l'espace local de la santé révèle la cohabitation de deux principaux modèles de santé autour desquels s'agrège une pluralité d'acteurs. II s'agit du " modèle biomédical » et du " modèle traditionnel ». Dans le modèle biomédical, on retrouve l'offre de services de santé du secteur public et du secteur privé ${ }^{6}$ et celle du secteur non officiel dit "informel ». Quant au modèle traditionnel, il propose les services des Gegè " cliniciens ", des Khamsi "thérapeutes sans thérapeutique " et des Gèkè " thérapeutes magiciens " (Lado et Fosso, 2010). Le recours à chaque modèle est fonction des rapports individuels et collectifs à la maladie et à l'offre de soins, construits au fil de l'histoire. Dans l'ensemble, le pluralisme thérapeutique est une réalité qui se mesure à travers la complexité des itinéraires thérapeutiques des patients. C'est dans ce contexte marqué du "soigner au pluriel " (Benoist, 1996) qu'est mise en œuvre la délivrance des ACT.

La collecte des données a été faite aux moyens d'entretiens et d'observations. Pour les entretiens, le concept de "groupe stratégique » (Olivier de Sardan, 2008) a été particulièrement fécond pour avoir révélé les jeux d'acteurs et d'intérêts autour de la délivrance des ACT, lesquels jeux donnent sens au concept " $d^{\prime}$ arène locale ${ }^{7}$. Les entretiens ont été menés avec trois responsables de l'administration locale, douze personnels de santé, six représentants locaux (élus et leaders associatifs), deux vendeurs en pharmacie d'officine, quatre vendeurs des «pharmacies de la

${ }^{5}$ Données du District de santé de Bandjoun de 2012.

${ }^{6} \mathrm{Au}$ moment de nos enquêtes Bandjoun comptait trois hôpitaux privés : I'hôpital de l'Eglise évangélique du Cameroun de Mbo, l'hôpital Ad lucem de Banjoun et le centre de santé intégré catholique de Semto. On y retrouvait également une pharmacie d'officine.

${ }^{7}$ Le recours à ce concept $d^{\prime}$ " arène " s'inscrit dans la dynamique des travaux déjà réalisés par J.P. Olivier de Sardan (2003a : 24) pour qui l'arène " est un lieu de confrontations concrètes d'acteurs sociaux en interaction autour d'enjeux communs ». Il précise qu'il s'agit d'un "concept souple, dont l'extension et la forme varient selon les contextes et les thèmes d'enquête. Sa valeur est avant tout exploratoire ". II convient tout de même de souligner que c'est d'abord chez Bailey (1969), qui définit l'arène comme un espace social de confrontations et d'affrontements du « jeu » politique national et local, qu'émerge ce concept. À Bandjoun, la délivrance des ACT s'opère dans un espace de " mise en scène " des " tactiques » d'acteurs qui font de cette action publique l'objet de « confrontations » d'intérêts. 
rue " (Jaffre, 1999) et dix relais communautaires. Au final, notre argumentaire s'articule autour des pratiques et logiques des acteurs locaux de la prescription et de la délivrance des ACT, ainsi que du positionnement des ACT sur le marché local des antipaludéens.

De l’introduction aux modes de délivrance différenciés des ACT

\section{De l'introduction des ACT au Cameroun...}

Les ACT qui arrivent sur le marché international des antipaludéens au début des années 2000 résultent d'un processus d'invention dont il convient de retenir quelques dates importantes. En 1972 l'artémisinine, présente dans la pharmacopée traditionnelle chinoise ${ }^{8}$ depuis 2000 ans, est isolée par extraction du principe actif en laboratoire. Au cours des années 1980, une équipe de I'Institute of Microbiology and Epidemiology de l'Academy of Military Medical de Pékin invente la combinaison associant les molécules artémether et luméfantrine. Cette combinaison est enregistrée en Chine en 1992. Par ailleurs, en 1991 l'artésunate est associée à la méfloquine pour le traitement du paludisme en Asie du Sud-Est. À la suite des progrès de la recherche sur les associations thérapeutiques destinées au traitement du paludisme, I'OMS recommande en 1998 I'utilisation de combinaisons d'antipaludéens. En 1999, arrive le Coartem (), une association d'artéméther et de luméfantrine. Le Coartem (C) est la première ACT à " dose fixe " ou " co-formulée " ${ }^{9}$, une invention chinoise sous brevet du laboratoire suisse Novartis (Orsi et Zimmermann, 2014).

\footnotetext{
8 L'artémisinine est produite à partir de la plante chinoise appelée kingaoushu. Dans la filière de la pharmacopée traditionnelle de Bandjoun, la présence de cette plante n'est pas signalée.

${ }^{9}$ Les ACT « co-formulées » se présentent sous la forme d'un comprimé unique associant deux molécules. Les " doses fixes " se distinguent des "doses séparées » dont les molécules sont produites séparément. L'approche de production de "doses séparées " a longtemps été pratiquée pour les associations antirétrovirales (ARV) car les molécules n'étaient pas libres d'usage et les brevets des molécules étaient détenus par des firmes pharmaceutiques différentes. Or, lorsque les ACT arrivent sur le marché, les molécules qui les composent sont libres et peuvent donc être copiées par tout producteur, ce qui va faciliter la production de « doses fixes ".
} 
En avril 2001, dans son rôle de "prescripteur global ", I'OMS recommande quatre combinaisons pour le traitement du paludisme ${ }^{10}$ :

- $\quad$ artésunate + amodiaquine (AS-AQ) ;

- $\quad$ artésunate + méfloquine (AS-MQ) ;

- $\quad$ artémether + luméfantrine $(\mathrm{AL})$;

- $\quad$ artésunate + sulfadoxine- pyriméthamine (AS-SP).

Tenant compte du fait que ces combinaisons ont des qualités différentes selon les situations épidémiologiques locales, I'OMS recommande pour l'Afrique soit l'AL, soit I'AS-AQ, soit les deux en traitement de première ligne ${ }^{11}$.

Au Cameroun, des années 1950 au début des années 2000 la lutte contre le paludisme s'est basée sur l'utilisation de la chloroquine pour le traitement des accès palustres simples. Durant cette période, ce médicament a également servi pour la chimioprophylaxie (prévention) chez les enfants et les femmes enceintes, populations à risque ${ }^{12}$. Avec l'échec thérapeutique de la chloroquine lié à l'apparition et au développement des résistances à cette molécule, les autorités du ministère de la Santé ont encouragé son retrait du marché camerounais en $2002^{13}$, engageant le

10 Il est à noter que depuis 2001 de nouvelles combinaisons ont été introduites sur le marché. À ce jour, 33 ACT ont obtenu la pré-qualification de l'OMS. Voir sur ce sujet: apps.who.int/prequal/

${ }^{11}$ Voir : who.int/entity/malaria/am_drug_policies_by_region_afro/en/index.html

12 Les cas graves qui résultent de l'inefficacité ou de l'absence de traitement des accès palustres simples sont traités par la quinine qui reste efficace.

${ }^{13}$ Les premiers échecs thérapeutiques de la chloroquine au Cameroun apparaissent durant les années 1980 dans la partie méridionale du pays (Brasseur, Druilhe et al., 1986). Par ailleurs, il convient de préciser que, de 1985 à 2002, la chloroquino-résistance connaît une expansion spatiale et pharmacologique sur l'ensemble du Cameroun. Le seuil de résistance défini par les protocoles de l'OMS, passé de 25 à $10 \%$, a été largement dépassé par la chloroquine qui va atteindre $65 \%$ dans la région côtière de la partie méridionale du Cameroun au début des années 2000. 
pays vers une période transitoire ${ }^{14}$. L'amodiaquine est alors utilisée en " première ligne ${ }^{15}$, c'est-à-dire prescrite en première intention pour la prise en charge des cas d'accès palustre simple, et la sulfadoxinepyriméthamine $(\mathrm{SP})^{16}$ en traitement préventif intermittent (TPI) chez les femmes enceintes.

Cette période transitoire a permis au Cameroun d'organiser, sous l'impulsion de l'OMS, le passage du traitement du paludisme par les monosubstances (chloroquine et amodiaquine) au traitement par les associations médicamenteuses (ACT). C'est par la décision ministérielle du 6 mai $2006^{17}$, qui elle-même fait suite aux résolutions des travaux de l'« Atelier de consensus national sur le traitement du paludisme " tenu en janvier 2004, que les mono-substances ont été définitivement retirées du marché camerounais et remplacées par les ACT.

Ainsi, la combinaison AS-AQ, qui durant nos investigations dans le district de santé de Bandjoun était disponible sous la forme de Falcimon, est adoptée comme médicament de première ligne par I'" Atelier de consensus national sur le traitement du paludisme ". Ce choix de l'AS-AQ n'a pas été exclusivement motivé par les arguments de différenciation selon les caractéristiques épidémiologiques du pays. En réalité, les arguments relatifs à l'implantation des fournisseurs - circuits commerciaux -, à l'histoire de l'offre - antériorité du Coartem premier ACT disponible sur le marché camerounais depuis 1999 - et surtout au lobbying ${ }^{18}$ parfois agressif (Fosso,

${ }^{14}$ Cette période transitoire a été recommandée par l'OMS, en attendant les conclusions des recherches sur les "évidences scientifiques » de l'efficacité des combinaisons thérapeutiques pour le traitement du paludisme.

${ }^{15}$ Les médicaments de "première ligne » sont sélectionnés sur la base de normes officielles en fonction de la prévalence de la maladie, de l'innocuité du médicament, de son efficacité et d'une comparaison des rapports coût-efficacité.

${ }^{16}$ Il faut noter que la SP, association de deux médicaments bloquant à divers niveaux la synthèse de l'acide folique chez le parasite, est considérée comme monothérapie.

${ }^{17} \mathrm{Cf}$. Décision n 0207 MINSANTE du 06 mai 2006 fixant les modalités de retrait du marché de certains médicaments antipaludiques.

18 Dans un précédent article (Fosso, 2013), nous montrions que lors de l'Atelier de 2004 chargé de proposer l'ACT de première ligne à adopter par le Cameroun, le lobbying des firmes 
2013) ont fortement pesé sur le choix de l'AS-AQ par les autorités du ministère de la Santé du Cameroun.

... à une délivrance des ACT à "prix différencié » dans un espace sanitaire

Afin de faciliter l'accès aux ACT, l'OMS signe en 2001 un accord exclusif de dix ans avec la firme pharmaceutique Novartis pour l'approvisionnement du marché public en Coartem à prix de sortie usine ne dégageant ni perte ni profit (entre 0,90 et $2,40 \$$ ). Le compromis implicite de cet accord entre I'OMS et le laboratoire Novartis est de laisser libre la fixation des prix du même produit sur le marché privé (Orsi et Zimmermann, 2014). Toutefois, le quasi-monopole du marché public par Novartis est contesté dès 2005 par l'agrément donné par le Fonds mondial aux firmes pharmaceutiques indiennes Ajanta en fin 2005 et Cipla en 2006 pour la production de l'AL, d'une part, et le partenariat entre l'Initiative for Drug Neglected Diseases ( $\mathrm{DnDi}$ ) et la firme pharmaceutique française Sanofi-Adventis pour la production de l'AS-AQ en 2006, d'autre part ${ }^{19}$. Ainsi s'amorce l'ère d'une stratégie globale de lutte contre le paludisme basée sur la politique du « prix différencié » entre marché public et privé. Sur le marché public, les prix des produits sont négociés entre l'OMS, les bailleurs de fonds internationaux et les firmes pharmaceutiques, tandis que le marché privé est libéralisé et peu régulé. Ce compromis positionne les grandes firmes pharmaceutiques internationales comme principales productrices d'ACT et n'accorde guère de place aux capacités endogènes de production des firmes pharmaceu-

pharmaceutiques a fortement influencé le choix I'AS-AQ. La principale firme qui soutenait I'AS-AQ avait fait valoir son réseau relationnel ancien dans le secteur de la santé au Cameroun, atout dont ne disposait pas Novartis propriétaire du Coartem.

${ }^{19}$ Sur les prix des ACT du marché public, il faut préciser que, sortie usine, l'AL coûte plus cher que l'AS-AQ. De 0,36 à $1,28 \$$ pour l'AL et de 0,24 à $0,75 \$$ pour l'AS-AQ en 2010 , selon le Global Price Reporting Mechanism for HIV, tuberculosis and malaria géré par l'OMS. 
tiques des pays endémiques, en particulier ceux d'Afrique subsaharienne dont le Cameroun ${ }^{20}$.

Au Cameroun, le Programme national de lutte contre le paludisme (PNLP) est chargé de l' « implémentation » de la politique de délivrance des ACT du marché public. À cet effet, la délivrance des ACT s'organise dans le circuit hospitalier. Dans ce dispositif du marché public des ACT, la Centrale nationale d'approvisionnement en médicaments essentiels et consommables médicaux (CENAME) et les centres d'approvisionnement régionaux en produits pharmaceutiques (CAPR) sont chargés de l'approvisionnement des hôpitaux publics et privés en ACT qui à leur tour les délivrent aux patients. Les ACT du marché public sont gratuites pour les enfants de moins de 5 ans et vendues à un prix réduit pour le reste de la population ${ }^{21}$. II est à noter que, si dans les pharmacies des hôpitaux publics on retrouve exclusivement I'ACT de première ligne, les hôpitaux privés eux ont la liberté de disposer d'autres ACT en plus de celle de première ligne.

Dans le secteur du marché privé, où est appliquée la libre fixation des prix des ACT, opèrent principalement les pharmacies d'officine et les "pharmacies de la rue ». Les officines pharmaceutiques, selon la norme officielle, s'approvisionnent en fonction des autorisations de mise sur le marché, appelées "visas " au Cameroun. Les "visas " sont délivrés par la direction de la Pharmacie et du Médicament (DPM) du ministère de la Santé publique. Dans ce dispositif légal, interviennent les firmes pharmaceutiques qui, par la médiation des délégués médicaux, font la promotion de leurs médicaments. En fonction de la demande sur le marché local, les médicaments sont commandés auprès des laboratoires pharmaceutiques par les grossistes officiels chargés de l'approvisionnement des pharmacies. Toutefois, il est à noter que de nombreuses pharmacies s'approvisionnent

\footnotetext{
${ }^{20} \mathrm{Au}$ Cameroun on retrouve à titre d'illustration le laboratoire Biopharma implanté à Douala. Toutefois, il est à noter que les capacités de production des firmes pharmaceutiques indiennes sont quasiment une exception.

${ }^{21}$ L'exemption totale de paiement pour les enfants de 0 à 5 ans est appliquée depuis 2010 . Quant à l'exemption partielle de paiement, elle est en cours depuis 2007 grâce aux financements du Fonds mondial.
} 
directement auprès des laboratoires à l'étranger ${ }^{22}$, sans recourir aux grossistes nationaux.

Quant aux "pharmacies de la rue " considérées comme "illégales " ou " clandestines " par le ministère de la Santé, on y retrouve des médicaments d'origines diverses, bien que l'essentiel des médicaments disponibles dans ce secteur provienne de grossistes "clandestins " nationaux. Ces grossistes, cheville ouvrière d'un système aux méandres insaisissables, s'approvisionnent auprès de laboratoires pharmaceutiques légaux et illégaux, pourvoyeurs d'antipaludéens aussi bien de qualité et d'origine contrôlée que sous-dosés et d'origine douteuse (Basco, 2004).

$\mathrm{Au}$ demeurant, le Cameroun se retrouve en situation d' « implémentation " d'une politique du " prix différencié » des ACT organisée à l'échelle mondiale et qui pose le problème de son application au niveau local où la circulation des médicaments entre les différents circuits est une pratique courante et ancienne (Van Der Geest, 1987). La délivrance des ACT du marché public, principal enjeu de la stratégie mondiale de lutte contre le paludisme, mobilise aussi bien les hôpitaux publics que privés. La véritable interrogation est de savoir si avec cette approche de délivrance des ACT du marché public suivant les principes de " non exclusivité » et de " non rivalité ", le circuit hospitalier va réussir à " capter » une "clientèle » habituée à s'approvisionner dans le marché privé des pharmacies d'officine et de la rue ${ }^{23}$.

\section{Le circuit hospitalier et la délivrance des ACT du marché public}

Dans la localité de Bandjoun, les deux marchés des ACT (public et privé) sont présents. Autour de ces marchés gravitent trois circuits de délivrance

\footnotetext{
${ }^{22}$ Cette modalité d'approvisionnement s'effectue souvent de manière frauduleuse, sans que l'on puisse s'assurer de la qualité des médicaments fournis.

${ }^{23}$ L'« affaire » de la réimportation vers l'Europe des antirétroviraux destinés au marché sénégalais, rendue publique en octobre 2002 (Taverne et Egrot, 2015), illustre bien les difficultés de la mise en œuvre de la politique du " prix différencié » dans le contexte actuel de circulation relativement aisée des médicaments.
} 
aux modes opératoires distincts. II s'agit du circuit hospitalier pour le marché public et des circuits des pharmacies d'officine et de la rue pour le marché privé.

Le dispositif hospitalier sur lequel s'appuie la délivrance des ACT du marché public au Cameroun est principalement celui des hôpitaux publics. Les hôpitaux privés sont également impliqués, comme acteurs de second plan, afin de remédier aux limites du dispositif hospitalier public qui peine à couvrir l'ensemble du territoire national (Gruénais, 2001). Certes, à Bandjoun comme dans l'ensemble du pays, le réseau des hôpitaux publics a connu une expansion depuis la mise en œuvre de la " décentralisation fonctionnelle » du secteur de la santé amorcée en 1993. Toutefois, malgré l'accroissement de l'offre de soins des hôpitaux publics, la " qualité " des services n'a guère suivi (Gruénais, 2004). Ainsi, la population de Bandjoun recourt majoritairement aux services des trois hôpitaux privés confessionnels ${ }^{24}$, plus anciens et de meilleure réputation (Lado et Fosso, 2010). Dans cette localité, pour trouver un hôpital public " de proximité " sollicité par les patients, il faut se rendre au centre médical d'arrondissement (CMA) de Ndem-deng. Le second CMA, celui de Poumegne, ainsi que les CSI, sont régulièrement déserts ${ }^{25}$. C'est dans ce circuit hospitalier qu'interviennent les deux "groupes stratégiques » mobilisés pour la délivrance des ACT du marché public : les personnels hospitaliers et les relais communautaires.

\section{Le personnel hospitalier : des acteurs et des tactiques}

Le personnel hospitalier est concerné à deux niveaux du circuit de délivrance des ACT : dans un premier temps, la prescription du médicament par les médecins et les infirmiers; dans un second temps, la vente ou " don » gratuit des ACT par les gestionnaires des pharmacies des hôpitaux. Notons que l'ordonnance du prescripteur est le moyen exclusif d'accès aux ACT de la pharmacie de l'hôpital.

\footnotetext{
${ }^{24}$ Les hôpitaux les plus sollicités de cette localité sont le centre de santé catholique de Semto, I'hôpital Ad-lucem de Bandjoun et l'hôpital protestant de Mbo, tous du secteur privé.

${ }^{25}$ Cette configuration du paysage hospitalier de Bandjoun explique le choix de la collecte des données principalement au CMA de Ndem-deng et à l'hôpital Ad-lucem de Bandjoun.
} 
Lors de l'enquête menée au CMA de Ndem-deng et à l'hôpital privé Adlucem de Bandjoun, il est apparu que les prescriptions d'ACT sont prédéterminées par la situation économique des patients. Dans un cas comme dans l'autre, la précarité généralement déclarée par les patients ou postulée par les prescripteurs influence le choix de la molécule d'ACT. À ce niveau, il faut préciser que c'est la stratégie de " liberté de prescription » qui est en vigueur au Cameroun et le choix du traitement de première ligne n'est pas une obligation. Ainsi, le prescripteur n'est pas obligé de choisir l'ACT de première ligne (AS-AQ), bien qu'elle soit la seule disponible à la pharmacie de l'hôpital public. Le choix de I'AS-AQ en première intention par les prescripteurs, en apparence purement technique, résulte bien des logiques des acteurs nationaux et internationaux des choix des stratégies de lutte contre le paludisme au Cameroun (Fosso, 2013).

Dans les pharmacies des hôpitaux privés, qui ont la possibilité de diversifier leur offre d'ACT, on observe que l'offre est réduite au Falcimon (AS$A Q$ de première ligne) et au Coartem (AL). Le Coartem disponible provient de dons des partenaires internationaux de ces hôpitaux, qui le vendent à prix réduit ${ }^{26}$.

Compte tenu de la régularité des ruptures de stock d'AS-AQ du marché public et de l'ordre de préférence des ACT par les patients, la prescription de la molécule de première ligne n'est pas systématique. Aussi l'option de la prescription du dihydroartémisinine + pipéraquine-phosphate (DH-PP), une combinaison introduite en 2010 sur le marché camerounais sous les formes commerciales du Malacur ou du Duo-cotecxin, est faite pour les patients nantis, tandis que le choix de l'AL, sous la forme commerciale du Coartem, est très souvent fait en réponse aux sollicitations de patients habitués et satisfaits de son usage. En somme, les prescriptions varient selon que le patient est " pauvre » (AS-AQ), nanti (DH-PP) ou bien selon des exigences spécifiques (AL). Par ailleurs, le choix du soignant peut aussi porter sur des médicaments qui ne sont ni gratuits ni subventionnés. Ainsi, la prescription de quinine, en principe réservée aux cas graves mais dont le produit de la vente rentre dans les recettes de l'hôpital, est une pratique

${ }^{26}$ A titre d'exemple, le Coartem pour enfant disponible à l'hôpital Ad lucem de Bandjoun, offert par un de ses partenaires allemands, coûtait 500 francs CFA (0,89\$) en 2011. 
courante dans les formations hospitalières. Cette prescription hors normes est fréquemment justifiée par l'argument d'une rupture de stock d'ACT, très souvent fictive.

De plus, du fait notamment des frais de consultation et des examens à payer avant la délivrance des ordonnances et des médicaments ${ }^{27}$, les hôpitaux sont en général très peu sollicités par les usagers en premier recours pour le traitement du paludisme ${ }^{28}$. D'autant plus que, dans de nombreux cas, les examens médicaux exigés par les soignants lors des consultations sont peu en lien avec les symptômes que présente le patient ${ }^{29}$. Dans ces hôpitaux, le coût des examens est élevé et reste hors de portée des bourses d'une population majoritairement paysanne ${ }^{30}$. C'est seulement à la suite des échecs consécutifs à l'automédication faite à partir de traitements traditionnels ou de médicaments achetés dans les pharmacies de la rue, que les patients s'adressent aux hôpitaux (Lado et Fosso, 2010). Toutefois, dans ces hôpitaux, des formes de solidarité peuvent naître et imposer des options différentes au prescripteur (médecin ou infirmier). Sur la base de l'interprétation des manifestations symptomatiques et sans examen, il peut faire un " don » de la dose d'ACT nécessaire pour le traitement. Mais cela se fait rarement sans que les frais de consultation n'aient été préalablement payés. L'exception du « don » d'ACT sans que le patient n'ait payé

${ }^{27}$ Cette barrière du coût de la consultation reste un obstacle à l'efficacité de la politique d'exemption de paiement du traitement du paludisme mise en œuvre dans les pays africains (Touré et Sanogo, 2014).

${ }^{28}$ Sur la question des déterminants des modes de recours aux hôpitaux en cas de paludisme, se référer à S. Faye et al. (2004) et L. Lado et A. L. Fosso (2010). Globalement, ils sont multiples (représentations de la maladie, qualité de l'offre de soins dans les hôpitaux, gestion socioculturelle et économique de la maladie...) et ne se résument pas à la seule pauvreté.

${ }^{29}$ Une illustration de cette pratique de la prescription d'examens sans lien avec la maladie et qui découragent les patients est rapportée par L. Touré et Y.T. Sanogo (2014) au sujet du paludisme au Mali.

${ }^{30}$ Précisons que durant la période de nos investigations, les tests de diagnostic rapide (TDR) n'avaient pas encore été introduits dans ce district de santé. La disponibilité des TDR aurait pu contribuer à rendre davantage efficace la délivrance des ACT du marché public en réduisant les coûts et la durée des examens d'une part et en facilitant la prise en charge rapide des cas par le personnel soignant et les relais communautaires d'autre part. 
les frais de consultation ni les frais d'examens concerne des pratiques en marge de la norme officielle, dites "consultations familiales " ${ }^{31}$, et, dans une moindre mesure, les consultations d'enfants "de condition précaire ": "Quand je reçois certains enfants malades, je suis parfois obligé de donner le Falcimon pour rien (sans paiement des frais de consultation et sans examens) ", confie un professionnel de santé.

Consécutivement à l'étape de la prescription, il y a l'achat des médicaments à la pharmacie de l'hôpital pour ceux des patients (adolescents et adultes) qui disposent des possibilités financières. Dans les pharmacies des hôpitaux publics exercent des commis, une catégorie du personnel hospitalier choisie parmi les membres de la " communauté » de l'aire de santé à laquelle appartient la formation hospitalière. Ces commis sont recrutés par un conseil réunissant le dirigeant de l'hôpital et le comité de gestion dudit hôpital. En général, pour être choisi comme commis il suffit de savoir lire, écrire et d'appartenir au réseau relationnel (parents, amis et connaissances) des responsables en charge du recrutement ${ }^{32}$. Les commis sont rémunérés par le comité de gestion de l'hôpital à partir des recettes propres du centre, provenant du recouvrement des coûts. Ils doivent rendre des comptes au comité de gestion et aux dirigeants des formations hospitalières qui les emploient. Un commis peut être affecté au poste d'aide-soignant ou de gérant d'une pharmacie dans une formation hospitalière.

En tant que gérants des pharmacies, les commis sont des acteurs au quotidien de pratiques particulières autour des ACT. Ils font de leur espace de travail le cadre de déploiement de tactiques personnelles. La disponibilité des ACT gratuites ou à prix réduit devient, dans cet espace, un « objet de politique » en fonction des configurations des rapports entre le commis et l'utilisateur.

\footnotetext{
${ }^{31}$ Les consultations familiales sont des prestations délivrées gratuitement aux membres de la « famille élargie » du soignant.

${ }^{32}$ Dans les hôpitaux privés, en raison de l'impératif de rentabilité, le personnel est recruté sur la base de qualifications en gestion des pharmacies, ce qui n'est pas toujours le cas pour les hôpitaux publics.
} 
Ces configurations recouvrent trois registres. Dans le premier cas, du fait de la présence d'instances de contrôle et de supervision, le commis observe strictement les procédures et met les ACT à la disposition des usagers sans en tirer un quelconque bénéfice personnel ${ }^{33}$. Le deuxième registre est celui des " pratiques courantes", caractérisées par la mise en place d'un système de tarification différent des tarifs officiels. II en résulte que les ACT gratuites deviennent payantes et que celles subventionnées voient leur prix passer du simple au double. "Chaque commis fixe les prix comme il veut et du jour au lendemain le prix peut passer de 150 à 300 francs CFA (de 0,26 à 0,52 \$) » reconnaît un commis. Quant au dernier registre, il s'agit de celui de la rupture artificielle des stocks afin d'entretenir un circuit clandestin de vente intra et extra hospitalier. La stratégie consiste ici à déclarer une rupture des stocks afin de préempter les médicaments disponibles qui sont ensuite écoulés au " marché noir ". Ce " marché noir " opère aussi bien au sein même de l'hôpital qu'aux domiciles des personnels hospitaliers et dans le circuit des pharmacies de la rue où l'on retrouve, en vente libre, les ACT destinées aux pharmacies des hôpitaux. Une autre stratégie consiste à vider les stocks des formations sanitaires les plus exposées aux contrôles, pour approvisionner celles situées dans les zones enclavées, rarement contrôlées, où la manipulation des prix peut se faire sans grande crainte. Certes, on peut y voir une démarche vertueuse visant à réduire les inégalités spatiales d'accès aux médicaments, mais en réalité il s'agit d'une tactique de captation de la rente ${ }^{34}$.

Ces pratiques ne sont pas le seul fait des commis qui, en général, opèrent en " coaction " active ou passive avec le reste du personnel hospitalier. La précarité de l'emploi est invoquée pour justifier ces pratiques ancrées et relevant d'une certaine "culture professionnelle spécifique de la santé " éloignée des normes officielles (Olivier de Sardan, 2003). En fait,

\footnotetext{
${ }^{33}$ Nous avons pu observer cette modalité du travail des commis à l'occasion de la descente à I'hôpital de district de Dja' à Bandjoun d'une équipe de supervision du Programme national de lutte contre le paludisme (PNLP) venue de la capitale Yaoundé.

${ }^{34}$ Nous avons pu observer cette différence de prix des ACT entre le centre de santé de Sedembo, situé en périphérie, et l'hôpital de district de Bandjoun, au centre de la ville.
} 
les commis sont très souvent recrutés dans la masse des diplômés victimes de la crise de l'emploi, elle-même générée par la crise économique des années 1980 au Cameroun. Dans ce contexte, servir comme commis au sein des formations hospitalières s'inscrit dans une dynamique $d^{\prime}$ '« employabilité de crise » et les revenus mensuels, particulièrement bas, ne permettent guère de satisfaire les besoins primaires vitaux. Les données de l'enquête révèlent à ce sujet que les salaires mensuels de cette catégorie du personnel hospitalier oscillent entre 10000 et 15000 francs CFA (entre 16 et $23 €$ ), loin du salaire minimum qui était de 28216 francs CFA $(43 €)^{35}$ lors de notre enquête de 2009 à 2012.

Dans ce contexte, se servir de son espace professionnel afin d'arrondir les fins de mois devient une tactique quasi normée de survie. Dans leurs pratiques au quotidien, il est fort remarquable d'observer la collusion qui existe entre les soignants et les commis. L'interdépendance entre ces deux acteurs de la délivrance des ACT établit une " complicité " dans l'action, et I'un ne peut se servir sans servir l'autre. II est à relever que ce qui vaut pour les ACT l'est également pour les autres médicaments à grands enjeux économiques et sanitaires, en raison de leur forte sollicitation et de leur disponibilité dans les formations sanitaires. Les trajectoires individuelles de ces commis permettent de renseigner sur leurs rapports particuliers aux médicaments en général et aux ACT en particulier. Le cas de ce commis rencontré dans la pharmacie d'un hôpital de Bandjoun est assez illustratif :

"Je travaille ici depuis 8 ans (2003) et on m'utilise comme aidesoignante et vendeuse à la pharmacie. C'est l'ancien surveillant général de l'hôpital qui m'a trouvé ce travail. Avant mon arrivée ici, je n'avais jamais travaillé, pourtant j'ai le BEPC (diplôme du cycle secondaire). Je cultivais les champs avant et même maintenant parce que ce que je gagne ne suffit pas pour nourrir ma famille. Je vis seule avec ma mère et mes trois enfants. Ici à l'hôpital, lorsqu'il y a quelque chose (rente), je demande toujours qu'on me mette dedans pour me permettre de gagner un peu d'argent. C'est pour cela que j'étais relais communautaire et maintenant je suis agent recenseur et distributeur pour la campagne des moustiquaires. Nous ici, nous

${ }^{35}$ Ce salaire minimum appliqué au Cameroun est passé à 36270 francs CFA $(55,5 €)$ le 25 juillet 2014. 
n'avons pas le choix, on doit se battre pour s'en sortir. Si en vendant un remède tu peux aussi avoir quelque chose pour nourrir ta famille tu vas demander un peu plus aux patients. Avec les médicaments du palu c'est facile d'avoir un peu d'argent parce que ça passe beaucoup. " (Entretien avec un commis de l'hôpital de Ndem-Deng, Bandjoun le 26 août 2011)36

\section{Les " relais communautaires », servir et se servir des ACT}

Le profil de "relais communautaire " a été introduit au Cameroun dans le cadre de la politique de "santé globale ». Ces acteurs de la délivrance des services de santé sont des bénévoles sollicités à l'origine pour le suivi des personnes vivant avec le $\mathrm{VIH}$. Ils reçoivent une prime monétaire en compensation de leur engagement. Leur champ d'action a été élargi à d'autres " Programmes de santé » dont - pendant une courte période - la lutte contre le paludisme ${ }^{37}$. Ces relais existent donc en permanence mais ont des tâches officielles variables. De plus, bien que cette activité soit en principe secondaire pour ceux qui l'exercent, elle constitue la principale source de revenu de nombreux relais.

Dans le district de santé de Bandjoun, l'activité de relais communautaire dans le cadre du Programme de lutte contre le paludisme n'a été signalée qu'au courant du second semestre de l'année $2008^{38}$. Bien que

${ }^{36}$ Lorsque ce commis déclare au sujet des ACT " ça passe beaucoup ", cela se vérifie non pas par les ventes officielles de la pharmacie de l'hôpital, mais bien par la forte demande des antipaludéens en dehors du circuit hospitalier, elle-même liée à la forte morbidité palustre, autour de $40 \%$ dans ce district de santé.

${ }^{37}$ On peut y lire une logique mimétique fondée sur l'épidémiologie et faisant abstraction de toute considération socioculturelle de la maladie. Or, dans certains contextes, la prise en charge de la maladie dans une perspective normative biomédicale ne sied guère à toutes les catégories affectées, comme le démontrent les travaux de L. Vidal (1999) sur la transmission du sida en Côte d'Ivoire. Ce qui est efficace pour la lutte contre le sida ne l'est pas forcément pour la lutte contre le paludisme.

38 Il convient de préciser que, en 2009, le Programme national de lutte contre le paludisme va transférer cette tâche aux organisations à base communautaire (OBC). Toutefois, durant nos investigations dans le district de santé de Bandjoun, l'implication des $O B C$ dans la délivrance des ACT n'était pas effective. Le recours aux OBC pour une activité du PNLP est signalé à 
cette activité ait été éphémère, il apparaît que les relais se sont illustrés par des rapports particuliers aux ACT. Comme dans le cas des commis des formations hospitalières publiques, la faible compensation financière a généré des pratiques autres que celles pour lesquelles ils étaient sollicités. Un relais communautaire du quartier Tsela à Bandjoun révèle :

"La vente des ACT moins chères à domicile marchait bien, mais il n'y avait pas beaucoup de remèdes. Malheureusement, la vente à domicile n'a pas duré longtemps, pourtant les gens s'habituaient déjà. Après l'arrêt du programme, moi j'ai continué à acheter pour venir revendre au quartier, mais un peu plus cher qu'avant. Les gens savaient déjà que je suis un "docta du palu" et me demandaient le Falcimon ou le Coartem dès qu'ils avaient un problème. Jusqu'à présent, lorsque j'ai un petit capital, je pars à l'hôpital acheter les médicaments pour revendre au quartier. " (entretien avec Christophe, ancien relais communautaire du quartier Tsela à Bandjoun le 30 novembre 2011)

Ainsi, outre le problème de la "viabilité » des interventions dans le cadre des programmes de santé, se pose celui des " manières de faire " de ces acteurs de terrain. En effet, lorsque ces relais sont connus, nombre d'entre eux pratiquent l'autolégitimation en se faisant passer pour des " docta ", c'est-à-dire des professionnels de santé. La qualification temporaire de relais pour le traitement des cas de paludisme simple a servi de prétexte à la pratique permanente d'activités médicales pour lesquelles ils n'ont aucune compétence légale, la logique consistant à se servir de la brèche ouverte par la création de ce nouveau profil d'acteur de la santé pour satisfaire leurs besoins personnels. Les exemples sont légions de relais communautaires qui outrepassent leurs prérogatives en s'adonnant à la pratique de la pose de perfusions sans en avoir la compétence ${ }^{39}$, mettant en évidence le problème du contrôle de l'activité de ces " auxiliaires de santé » dont la formation se limite à quelques heures de séminaire.

l'occasion de la campagne nationale de distribution des moustiquaires imprégnées aux ménages de 2011.

${ }^{39}$ Nous avons pu observer ces pratiques des relais communautaires à Bandjoun dans le cadre de nos précédentes recherches de Master entre 2007 et 2009, période durant laquelle leurs activités étaient en cours dans ce district de santé. 
Pendant la courte période de leur action officielle dans le cadre de la lutte contre le paludisme, ces relais se sont par ailleurs illustrés comme des sources d'approvisionnement en ACT pour le marché des pharmacies de la rue. À Bandjoun, en 2008, l'abondance des ACT sur le marché durant la période d'activité des relais communautaires est une coïncidence qui ne relève guère du hasard ${ }^{40}$. Un relais communautaire justifie ainsi ses « manières de faire $"$ :

"Ici au village, lorsque nous allons dans les maisons, les gens nous posent toujours les problèmes qu'ils ont. Si tu viens pour la distribution du Mectizan ou les moustiquaires, les mamans vont te demander de les consulter ou de leur donner des remèdes que tu n'as pas. Lorsque tu dis que tu ne peux pas, on considère que tu es méchant, que tu gardes pour toi ou que tu veux que les gens partent dépenser à l'hôpital. Donc, à force d'être sollicité, tu finis par faire ce que tu ne dois pas faire, surtout si ça peut aider les gens. » (entretien avec Bernard, ancien relais communautaire du PNLP du quartier Sédembo à Bandjoun)

Il faut le reconnaître, cette situation n'est pas propre à Bandjoun ou au Cameroun. Une illustration de ce désir de légitimation des relais communautaires dans le champ de la santé au Sénégal est rapportée par S. Faye (2012). Etre relais communautaire s'inscrit dans une stratégie de professionnalisation et, au Cameroun comme au Sénégal, ces acteurs de santé ont des ambitions dont les enjeux, en termes d'emploi et de dynamiques de coopération ou d'autonomisation, ne sont pas toujours en accord avec ceux des interventions ponctuelles entreprises dans le cadre du PNLP.

La pharmacie d'officine, un « monopole » sur un marché concurrentiel

Le marché privé de la délivrance des ACT à Bandjoun est marqué par la présence de deux circuits: celui de la pharmacie d'officine et celui des

${ }^{40}$ Données des observations de terrain au marché de Bandjoun durant nos investigations de Master puis de Doctorat (2007-2014). 
pharmacies de la rue. Dans ce secteur, rappelons-le, la libre fixation des prix des ACT est la règle.

Le secteur de la pharmacie d'officine dans le district de santé de Bandjoun est un domaine d'activité dans lequel on observe un monopole du marché, du fait de la présence d'une seule pharmacie. Celle-ci exerce suivant le mode opératoire des commerces ordinaires du marché de Bandjoun, avec pour objectif de vendre afin de réaliser le meilleur chiffre d'affaire possible. De la rentabilité de la pharmacie dépendent la sécurité des emplois du personnel et la prospérité du propriétaire. Ce dernier, propriétaire d'une autre pharmacie dans une grande ville du pays, ne réside pas à Bandjoun. La pharmacie de Bandjoun est donc placée sous la responsabilité d'un gestionnaire dont le rôle se limite à la supervision des équipes de vente. Pour le fonctionnement de ce "commerce", point besoin de s'encombrer des compétences de pharmacien, seule une aptitude certaine en matière d'appât et de fidélisation de la clientèle est utile. Au sujet de l'activité de cette pharmacie, un employé confie :

"La pharmacie c'est d'abord le commerce. Comme il y a beaucoup le palu chez nous, les remèdes du palu passent (rapportent) plus que les autres. II faut savoir attirer les clients mais en faisant attention. Le problème avec la pharmacie ici c'est le prix des médicaments. Les ACT coûtent à partir de 2500 francs CFA $(3,81 €)$ pourtant là à côté, il y a une boutique où tu trouves les mêmes ACT à 500 francs $(0,81 €)$. Donc les gens préfèrent aller à la boutique, même les personnels de santé. Les gens n'ont pas d'argent et doivent se débrouiller pour se soigner. » (entretien avec un vendeur de la pharmacie de Bandjoun, le 16 novembre 2011)

En matière de rapport aux $\mathrm{ACT}$, les données de l'enquête indiquent que les vendeurs de cette pharmacie opèrent suivant deux modalités. Dans le premier cas, il est question de répondre à une demande exprimée par le client soit au moyen d'une ordonnance, soit verbalement. La seconde posture est celle de la suggestion et, dans ce cas, des propositions de médicaments sont faites à la clientèle. Dans un cas comme dans l'autre, l'enjeu commercial est prioritaire. Lorsqu'on interroge le niveau de connaissance des vendeurs sur les ACT, force est de constater que la maîtrise des molécules n'est pas toujours la chose la mieux partagée. De ce fait s'opèrent des pratiques de vente qui ne font guère la part entre les antipaludéens 
pour les cas simples - exclusivement les ACT - et ceux destinés aux cas compliqués - la quinine sous ses diverses présentations. Ces pratiques traduisent le fait qu'on est en présence d'une " officine sans pharmacien ".

Malgré la situation de monopole de cette pharmacie, la réalité de l'activité de vente des ACT révèle une certaine concurrence entre cette " officine sans pharmacien " et les "pharmacies de la rue ". Les pics de vente obéissent au rythme de la programmation bihebdomadaire des " jours du marché ${ }^{41}$ de Bandjoun, jour où affluent pharmaciens de la rue et clients de Bandjoun ainsi que des villages environnants. La position stratégique de la pharmacie, située à l'entrée du marché de Bandjoun, est révélatrice de son offensive sur le terrain d'opération habituel des " pharmacies de la rue ». Grâce à cela, elle réussit à se maintenir malgré la concurrence des pharmacies des hôpitaux et des " pharmacies de la rue ».

Les « pharmacies de la rue » en position dominante sur le marché privé

Les "pharmacies de la rue " constituent le second circuit, dit "informel $\aleph^{42}$, du marché privé de la délivrance des ACT à Bandjoun.

Au regard de son organisation et de son fonctionnement, ce circuit de vente des médicaments n'a quasiment rien d'informel, si ce n'est l'absence d'un agrément officiel. Parmi les acteurs de ce circuit, on trouve les tenanciers des épiceries et petits commerces qui proposent quelques médicaments aux côtés d'une variété d'autres produits de consommation, les vendeurs ambulants qui sillonnent marchés et quartiers sans point de

${ }^{41}$ La notion de "jour du marché " s'inscrit dans la logique traditionnelle d'organisation des activités hebdomadaires. Selon ce calendrier traditionnel, la semaine compte huit jours et chaque jour est dédié à une activité particulière et vitale pour le vivre ensemble : jours consacrés à la pratique des rituels traditionnels ou jours du marché. A Bandjoun, le marché est ouvert tous les jours mais deux "jours du marché ", de grande affluence, se tiennent par semaine à intervalle de 72 heures, selon le calendrier traditionnel.

${ }^{42}$ Sur les qualificatifs de ce marché du médicament, les auteurs ne manquent guère d'inspiration. Il est ainsi qualifié de " parallèle ", de " clandestin ", d' « illicite " ou d' " informel » (Fassin, 1985 et 1986 ; Jaffré, 1999 ; Van Der Geest, 1987). 
vente fixe, les vendeurs à l'étal qui vont d'un marché à l'autre en fonction de la programmation des " jours du marché » et les vendeurs permanents du marché de Bandjoun qui disposent d'une boutique ouverte quotidiennement, exclusivement consacrée à la vente des médicaments. Les enquêtes de terrain ont montré que seuls les trois derniers profils sont impliqués dans la vente des ACT.

\section{Les ambulants limités par le capital}

Les vendeurs ambulants disposent d'une offre réduite de médicaments en raison de leur capital relativement faible. De ce fait, ils sont peu concernés par la vente des médicaments à prix élevé ${ }^{43}$. Les ACT faisant partie des " médicaments chers ", ils ne les livrent aux clients que sur commande. En général, ils réorientent plutôt les clients vers les vendeurs à l'étal ou vers la boutique du marché, principale concurrente de la pharmacie officielle, censés disposer d'ACT en permanence.

\section{Les occasionnels du " jour du marché »}

Chez les vendeurs bihebdomadaires, la tendance est à la vente des produits pharmaceutiques les plus sollicités par les patients. Sur ce marché où l'offre est fonction de la demande, on assiste à une stratégie de commercialisation du médicament déterminée par les utilisateurs. La part de I'incidence des décisions prises en amont dans le cadre d'une "politique de santé publique " ou d'une "politique du médicament » est faible et l'exemple des ACT en est la parfaite illustration. Les choix opérés en amont sont très peu intégrés dans les habitudes des utilisateurs en aval et les données de l'enquête montrent que la connaissance des ACT comme antipaludiques n'est pas spontanément affirmée par ces vendeurs occasionnels.

"Qu'est-ce que ça signifie ? " telle est la question récurrente lorsque ces vendeurs sont interrogés sur leur connaissance des ACT. C'est après

${ }^{43}$ Les prix des médicaments observés chez ces clandestins oscillent en général entre 25 francs CFA $(0,04 €)$ pour deux comprimés de paracétamol et 500 francs CFA $(0,81 €)$ pour la plaquette de quinine. 
explication que le qualificatif "ACT » est attribué à certains médicaments disposés sur la table qui sert de comptoir de vente mais, en général, les médicaments présentés ne sont pas toujours des ACT. Chez tous les vendeurs, on retrouve le Coartem et, dans une moindre mesure, le Falcimon. Toutefois, malgré le faible niveau de connaissance et d'information, en situation de conseil au patient se plaignant du paludisme, le premier choix du vendeur se porte sur le Coartem. Viennent ensuite l'amodiaquine et la quinine. La chloroquine, pourtant interdite à la vente au Cameroun, et la sulfadoxine-pyriméthamine sont quant à elles conseillées comme des médicaments administrés en prévention du paludisme.

Une expérience dans le secteur de la vente des médicaments par un occasionnel du marché de Bandjoun est rapportée ci-dessous :

"Certains clients viennent solliciter des conseils, d'autres viennent avec leurs idées. Mais, ce qui compte ici c'est de donner au client ce qu'il veut. Lorsque les gens viennent avec leurs idées, si tu te mets à donner des leçons, tu ne vas rien vendre ! [...] Moi j'ai une licence en biologie, j'ai été instituteur puis directeur d'une école catholique avant d'entrer dans le marché des médicaments il y a six ans. Je peux donc dire que je connais les remèdes. Mais comme les choses évoluent beaucoup, j'essaie de m'informer sur les nouveautés comme les ACT. Je ne vends pas beaucoup comme les autres, surtout ceux qui sont au bord de la route, parce que je fais attention à ce que je donne aux clients. Pour moi, il n'y a pas que l'argent qui compte. " (entretien avec Jean au marché de Bandjoun le 24 août $2012)^{44}$

Le rapport aux ACT de ces vendeurs est assez variable et fortement déterminé par leur niveau d'études. II est ainsi apparu lors de l'enquête que plus le vendeur est instruit, meilleure est sa connaissance des antipaludéens en général, et des ACT en particulier. Toutefois, ces vendeurs plus instruits et capables de porter un regard critique sur la "qualité " des médicaments en général et des antipaludéens en particulier, pèchent par leur tendance à contredire les clients qui, en général, viennent acheter le

${ }^{44} \mathrm{Ce}$ vendeur est le seul parmi les occasionnels du marché de Bandjoun à avoir une certaine connaissance des ACT. 
médicament qui, de leur point de vue, est le plus efficace. II n'est donc pas surprenant que cette catégorie ne réalise pas les mêmes ventes que les vendeurs pour qui seul compte le besoin énoncé par le client et qu'il faut satisfaire à tout prix.

Sur le marché de Bandjoun, durant toute la période de l'investigation de terrain, nous avons constaté la présence systématique de onze vendeurs à l'étal lors des " jours du marché ". Tous disposent d'un point de vente fixe et une table sert à l' " exposition des médicaments $»^{45}$. Ils sont sollicités pour le traitement des cas de paludisme simple et compliqué. Les approches sont fonction de la catégorie de la clientèle. Ainsi, ces vendeurs distinguent la clientèle des "connaisseurs" de celle des "ignorants". Si les premiers ne recourent pas aux conseils des vendeurs car ils disposent d'une ordonnance ou ont des " connaissances " sur les médicaments, les seconds sollicitent la consultation gratuite du vendeur. Dans ce cas, le vendeur est accessible et le client n'a pas à payer les frais de consultation ou d'examens avant la " prescription » du médicament. Le " diagnostic par intuition " est la pratique courante car aucun vendeur n'a de formation dans le domaine des soins. Le traitement se fait en général à partir d'une composition médicamenteuse comprenant antipaludéens et médicaments associés pour, dit-on, " une meilleure efficacité » (paracétamol et vermifuge).

L'approvisionnement en médicaments se fait en général sur les marchés des villes de Douala, Yaoundé et de Bafoussam. Lors des enquêtes, il a été signalé qu'avec l'essor du marché de la quincaillerie, les opérateurs de ce secteur assuraient également l'approvisionnement en médicaments en dissimulant ces derniers dans les cargaisons de matériaux de construction qu'ils font entrer dans le pays $^{46}$.

45 II convient de préciser que sur ces tables sont en général exposées des boîtes vides ou quelques tablettes de médicaments. Le gros du stock de médicaments est conservé dans des valises bien dissimulées ou dans des magasins à l'abri des expéditions punitives visant à " éradiquer » ce mode de délivrance des médicaments.

${ }^{46}$ La saisie record des stocks de médicaments avec au premier rang les antipaludiques au port de Douala en 2013 confirme cette donnée de terrain collectée plus d'un an avant ce coup de poing des services douaniers. 


\section{Le permanent du marché de Bandjoun : être formel ou ne pas l'être?}

Les vendeurs permanents au marché de Bandjoun sont de véritables concurrents de la pharmacie d'officine. Dans cette catégorie exerçaient jusqu'en 2010 deux commerçants de grande renommée. Mais depuis la fin de l'année 2010, il n'en reste plus qu'un seul sur ce marché ${ }^{47}$.

Ce prestataire offre une gamme très diversifiée d'ACT. Son offre est localement reconnue comme étant quantitativement supérieure à celle de la pharmacie d'officine de Bandjoun - il est d'ailleurs bien connu que ce point de vente sert très souvent de recours pour cette dernière, ainsi que pour les hôpitaux. "On y retrouve presque toujours le médicament recherché " dit-on localement. Son propriétaire justifie ainsi son succès :

"Lorsque tu travailles bien tout le monde t'apprécie, même ceux qui peuvent être contre toi. Vous-même avez constaté que chaque fois que vous venez ici il y a toujours du monde. Certaines personnes viennent parfois ici juste pour la conversation et je suis toujours disponible pour tout le monde, la preuve est que je suis disponible pour vous chaque fois que vous venez, pourtant je pourrais avoir peur que vous me dénonciez à Yaoundé. " (entretien du 18 octobre 2011 au marché de Bandjoun)

Le positionnement sociopolitique et économique ${ }^{48}$ du propriétaire de ce point de vente permanent en fait un acteur avec qui il faut composer. À la pharmacie d'officine, il n'est pas considéré comme un « clandestin » au

\footnotetext{
${ }^{47}$ Le second vendeur permanent en boutique n'a pas pu se remettre de la destruction de ses stocks de médicaments en 2010 par les services de santé et les forces de l'ordre. En fait, ce dernier ne disposait pas d'appui politique fort comme son collègue qui a échappé aux destructions grâce à ses informateurs. Il faut préciser que depuis la décision ministérielle conjointe, ministère du Développement industriel et commercial et ministère de la Santé publique, du 10 août 1996, la traque de la vente illicite des médicaments est devenue un enjeu de santé publique au Cameroun, même si l'on observe que l'application est sujette à controverse.

${ }^{48}$ Le tenancier de ce point de vente est un membre influant de la branche locale du parti au pouvoir au Cameroun. Par ailleurs, il compte parmi les fortunes locales, ce qui lui confère une position dominante et une capacité à influencer les agents publics en charge de la lutte contre la vente illicite des médicaments.
} 
même titre que les autres prestataires des "pharmacies de la rue " et, comme toutes les boutiques du marché de Bandjoun, il est astreint au paiement des impôts et taxes. Son propriétaire reçoit ainsi régulièrement la visite des agents de la commune en charge du recouvrement des taxes.

"Tout le monde me connait ici à Bandjoun. Comme tous les commerçants, je paye mes taxes et c'est bien parce que je fais bien mon travail que même les autorités viennent acheter les remèdes chez moi. Vous avez vu certains venir cette semaine » (entretien du 16 octobre 2011 au marché de Bandjoun)

Dans son déploiement quotidien, le responsable de cette boutique accorde une grande importance au " dialogue " avec ses clients. Le rapport au client n'est pas celui de la simple vente d'un produit comme dans un commerce ordinaire. Cet autodidacte procède par suivi des prescriptions des médecins et des infirmiers ainsi que par adaptation en autoprescription au moyen des dictionnaires thérapeutiques ${ }^{49}$. Bien qu'il dispose d'une gamme d'antipaludéens assez variée, les médicaments les plus vendus sont le Coartem, la quinine et, dans une moindre mesure, le Falcimon. Il est remarquable d'observer l'absence des monothérapies interdites à la vente qui pourtant pullulent chez les vendeurs à l'étal. Un effort est ainsi fait en matière d'observation des directives officielles pour la vente des antipaludéens. Sur son rapport à la clientèle et la qualité des services, le responsable déclare ainsi :

"C'est vrai que je suis très populaire ici et ça aide dans le commerce. Moi j'écoute les gens, je donne des conseils et parfois lorsque quelqu'un n'a pas les moyens, je peux lui donner les remèdes qu'il payera après. Une chose très importante est que je m'assure toujours que mes médicaments sont de bonne qualité, et c'est surtout pour ça que mes affaires marchent » (entretien du 18 octobre 2011 au marché de Bandjoun)

Globalement, ce point de vente se distingue par une importante offre de produits pharmaceutiques, par son accessibilité et la légitimité socialement construite de son promoteur. Cette situation dénote un double ancrage marqué par des emprunts au dispositif légal et au circuit des phar-

${ }^{49}$ Dictionnaire thérapeutique Vidal, notamment. 
macies de la rue. Prend également forme dans cette boutique une synthèse du pluralisme médical à l'échelle locale. Celle-ci se traduit par la cohabitation d'une gamme variée d'antipaludéens provenant de laboratoires de l' " industrie biomédicale occidentale ", de l'offre thérapeutique indienne et chinoise et de l'offre de la « médecine traditionnelle » locale. À ce sujet le responsable rapporte :

" J'ai aussi développé de bonnes relations avec les laboratoires chinois qui m'invitent à participer à leurs conférences pour mieux connaitre leurs produits. La dernière fois, j'ai participé à un séminaire à Yaoundé organisé par les chinois. Voici leurs produits du palu bien positionnés au comptoir. Ils donnent les ristournes aux revendeurs et lorsque tu deviens un grossiste de leurs produits, ils t'offrent une voiture pour tes livraisons. Je peux dire que tout le monde trouve son compte chez moi. Si tu veux les remèdes traditionnels, chinois ou modernes, tu les trouveras chez moi » (entretien du 24 août 2011 au marché de Bandjoun)

De tous les antipaludéens disponibles, on observe une préférence pour le Coartem en première intention. Toutefois, les médicaments traditionnels et chinois sont aussi de manipulation fréquente. Dans le cadre de sa collaboration avec les laboratoires indiens et chinois, ce vendeur déclare ainsi avoir été formé à la connaissance et à la vente de leurs produits. Les firmes pharmaceutiques occidentales n'ont quant à elles pas de contact officiel avec le propriétaire de cette boutique. Cependant, l'existence de contacts informels avec des délégués médicaux de ces firmes occidentales est envisageable, du fait de la présence, dans les stocks du magasin, de médicaments estampillés " échantillon médical gratuit » dont disposent les délégués médicaux pour la promotion des spécialités des firmes qu'ils représentent.

En somme, le " marché » de la délivrance des ACT à Bandjoun est marqué par la pluralité des acteurs qui interviennent suivant des logiques spécifiques. Malgré la politique d'exemption totale et partielle du paiement des ACT pratiquée dans le circuit hospitalier, le secteur privé a réussi à se positionner comme acteur de premier plan de la délivrance des ACT à Bandjoun. 


\section{Les ACT sur un marché concurrentiel}

La réalité du marché des $\mathrm{ACT}$ à Bandjoun est révélatrice de pratiques concurrentielles qui se manifestent sous deux formes : inter antipaludéens et intra ACT.

\section{Une concurrence entre les antipaludéens}

La concurrence entre les antipaludéens concerne l'ensemble des thérapeutiques du paludisme, dont les ACT. À Bandjoun, l'avènement des ACT comme traitement exclusif du paludisme simple n'a fait qu'accroître l'offre d'antipaludéens. Bien que le marché des médicaments du paludisme simple, du point de vue officiel, soit uniquement réservé à ces combinaisons thérapeutiques, les retours de terrain révèlent qu'on est loin de cette exclusivité. En effet, au côté des ACT, on retrouve l'offre thérapeutique " traditionnelle " et les monothérapies, qu'elles soient interdites à la vente ou administrées en "mésusage ". Afin de cerner le positionnement des ACT sur ce marché concurrentiel des antipaludéens, il importe de procéder à une analyse par circuit de délivrance des médicaments.

La concurrence entre les antipaludéens est davantage notable dans le modèle biomédical. Elle prend diverses formes, qu'il s'agisse du marché public ou du marché privé. Comme nous l'avons vu, dans le circuit hospitalier, l'offre d'antipaludéens n'est guère exhaustive pour les accès palustres simples. On retrouve en principe l'ACT recommandée - en l'occurrence la combinaison AS-AQ sous la forme de Falcimon - dans les hôpitaux publics, tandis les hôpitaux privés délivrent un AL - le Coartem - en plus de I'ACT de première ligne. Par ailleurs, dans ces hôpitaux publics et privés, malgré la norme officielle qui prescrit la délivrance des ACT en traitement des accès palustres simples, ceux-ci sont mis en concurrence avec la quinine destinée en principe aux cas de paludisme compliqué. En général, l'argument de la rupture des stocks d'ACT est mobilisé comme justificatif et la quinine est prescrite, malgré son coût élevé ${ }^{50}$.

${ }^{50}$ Les personnels soignants $n$ 'hésitent pas à prescrire la quinine pour les accès simples, malgré son prix élevé dans les pharmacies d’hôpitaux, de 300 à 700 francs CFA (0,53 à 1,25\$), par 
À la pharmacie d'officine, la tendance est au respect de la liste officielle des médicaments autorisés à la vente par la direction de la Pharmacie et du Médicament du ministère de la Santé. Toutefois, il apparaît que le recours à cette pharmacie est, en général, une modalité de la pratique de l'automédication : les clients, sans ordonnance, vont à la pharmacie pour s'approvisionner en antipaludéens. Il en résulte qu'en raison de l'absence d'un véritable pharmacien, les vendeurs de cette " officine sans pharmacien " proposent indistinctement des médicaments destinés tant aux cas de paludisme simple qu'aux cas de paludisme compliqué. Entre les ACT et les différentes spécialités de quinine, aucune véritable distinction n'est faite, seul compte l'enjeu de l'atteinte de la rentabilité financière par les vendeurs.

Dans le circuit des pharmacies de la rue, la disponibilité des ACT est fortement déterminée par la demande. La situation du marché de Bandjoun révèle que les ACT se noient au milieu d'une gamme variée d'antipaludéens qui intègre les monothérapies ainsi que les « médicaments chinois ", dont la ou les molécules sont en général inconnues du ministère de la Santé car ils sont souvent introduits soit comme des compléments alimentaires, soit par des circuits qui échappent au contrôle des autorités sanitaires. De la présence de cette gamme variée d'antipaludéens, trois constats se dégagent : (1) les ACT sont très faiblement représentées et le Coartem est la principale, voire la seule ACT connue et vendue; (2) les monothérapies de diverses origines sont largement majoritaires et représentent les principales ventes d'antipaludéens ; (3) la chloroquine, malgré l'interdiction de ce médicament par les autorités camerounaises, existe à nouveau sur le marché alors qu'en 2009 nous n'avions pas observé la présence de ce médicament.

\section{Une concurrence entre les ACT}

La concurrence entre les ACT sur le marché se décline en termes de partage des parts de marché parmi les combinaisons disponibles tant dans le circuit hospitalier que dans le marché privé. Dans le circuit hospitalier, un

rapport aux ACT gratuites ou vendues entre 140 et 250 francs CFA, soit 0,25 à $0,44 \$$, dans les mêmes pharmacies. 
constat se dégage : si l'AS-AQ a le monopole dans les hôpitaux publics, cette position est contestée par l'AL dans les hôpitaux privés. En effet, si les premiers sont astreints au suivi rigoureux de la délivrance de l'AS-AQ, les hôpitaux privés peuvent proposer une offre d'ACT plus diversifiée. De ce fait, on retrouve très souvent dans les hôpitaux privés, outre l'AS-AQ du marché public, la combinaison AL sous la forme du Coartem. Du fait de sa provenance (" dons " de partenaires internationaux), le prix de cette ACT n'est pas celui pratiqué sur le marché privé. Par ailleurs, ce mode d'approvisionnement en Coartem place l'AL en position dominante dans les hôpitaux privés - qui sont aussi les plus sollicités. L'AL représente donc la majorité des ventes d'ACT du circuit hospitalier à Bandjoun. Cette position dominante de l'AL observée au niveau local se confirme au niveau du marché public mondial (Orsi et Zimmermann, 2014).

Sur le marché privé des ACT, la concurrence est plus ouverte. Elle ne reflète pas uniquement un duel entre I'AS-AQ et l'AL mais prend la forme d'une concurrence multi-combinaisons qui, en plus des précédentes molécules d'ACT, met en scène toutes celles qui sont homologuées par la direction de la Pharmacie et du Médicament. Du fait de cette concurrence, la combinaison dihydroartémisinine + pipéraquine phosphate (DH-PP), ACT la plus coûteuse connue sous les noms commerciaux de Malacur et Duocotexin, est la plus sollicitée, devant la combinaison $\mathrm{AL}^{51}$.

Globalement, de la situation du marché concurrentiel des ACT, il apparaît que l'AL et la DH-PP sont les combinaisons les plus sollicitées. Cette préférence est révélatrice d'un mode de consommation des ACT qui n'est guère en accord avec les recommandations et les directives officielles en matière de prise en charge des cas de paludisme simple. Cette contradiction est révélatrice d'une certaine déconnexion entre le niveau de prise

\footnotetext{
${ }^{51}$ À titre indicatif, les ventes du mois de septembre 2011 de la pharmacie d'officine de Bandjoun révèlent que 41 doses de DH-PP ont été vendues contre 26 doses d'AL et 8 doses d'AS$A Q$. Dans le secteur des pharmacies de la rue où les médicaments se vendent au comprimé ou à la tablette selon les capacités financières des clients, il est difficile de fournir des chiffres comparatifs semblables à ceux de la pharmacie d'officine. Toutefois, nous avons relevé chez trois vendeurs sur étal durant la période du 20 septembre au 20 décembre 2011 la vente de 82 tablettes de Coartem, 32 tablettes de Falcimon, 76 tablettes de Fancida (SP), 604 comprimés de Quinine, 394 comprimés de Chloroquine et 202 comprimés d'Amodiaquine.
} 
des décisions et le niveau opérationnel en matière de politique du médicament, du moins dans le domaine de la lutte contre le paludisme simple. Même si l'automédication est un facteur clé dans les rapports individuels aux ACT, elle seule ne détermine pas l'ordre de préférence de ces combinaisons thérapeutiques. Ici interviennent également les ordonnances médicales qui, dans de nombreux cas, indiquent comme antipaludéens des molécules autres que l'AS-AQ. De l'avis des prestataires de soins du district de santé de Bandjoun, le Duocotexin et le Malacur sont ainsi les antipaludéens les plus efficaces et à meilleure innocuité (moins d'effets secondaires).

\section{Conclusion}

La délivrance des ACT à Bandjoun à l'Ouest du Cameroun met en scène des réseaux de prestataires, localement construits, qui mobilisent les divers acteurs déjà impliqués dans la délivrance des médicaments du marché public et du marché privé. Malgré la " position dominante » d'acteur de la délivrance des ACT conférée au secteur hospitalier dans le cadre de la mise en œuvre de la stratégie mondiale de lutte contre le paludisme, force est de constater que cette position est davantage postulée que véritablement effective sur le terrain.

Par ailleurs, l'analyse des pratiques au quotidien de l'ensemble des acteurs de la délivrance des ACT à Bandjoun dévoile des interdépendances fortes. Il en résulte une co-délivrance dans une configuration polymorphique en constante renégociation, qui révèle les écarts entre la norme officielle, "prescrite " par l'OMS dans le cadre de sa "Stratégie globale " de lutte contre le paludisme, et les pratiques réelles des acteurs de terrain $^{52}$. En fonction de leurs intérêts particuliers, ces derniers déploient des tactiques de contournement des protocoles officiels et créent leurs propres protocoles afin précisément de tirer profit au maximum de la politique du prix différencié des ACT.

52 II est ici question des "normes pratiques» (Olivier de Sardan, 2003b) qui régulent les rapports entre les acteurs de la délivrance des ACT. 
Il importe de relever que ce qui se joue localement à Bandjoun autour de la question plus large de l'application des politiques globales de santé à l'échelle locale n'est pas une spécificité de ce terrain d'étude. Les conclusions de Touré et Sanogo (2014), au sujet de la situation malienne, montrent que l'échec de la politique de gratuité de la délivrance des ACT par les services hospitaliers est également lié aux pratiques des professionnels de santé. Ceux-ci s'illustrent par la pratique de la rétention de l'information, qui induit un déficit de communication autour de cette politique, ainsi que par la production de ruptures de stock artificielles et donc " une disponibilité aléatoire des intrants gratuits dans les formations sanitaires". On retrouve par ailleurs, une " apparence de gratuité » ainsi que la non confirmation biologique du diagnostic clinique malgré la disponibilité, dans le cas du Mali, des tests de diagnostic rapide.

Les pratiques analysées à Bandjoun dénotent d'un échec du passage du global au local de la politique de délivrance à prix différencié des ACT. Cet échec s'explique par la logique essentiellement économique qui sous-tend cette politique globale initiée par l'OMS et basée sur la rencontre naturelle entre une offre et une demande. Or le secteur de la santé en général, et celui du médicament en particulier, se distingue par le fait que le " marché » est socialement construit et donc localement déterminé.

Bien que l'on puisse voir dans l'approche de l'OMS une posture vertueuse lorsqu'elle s'érige en " prescripteur global » organisant le marché public des ACT afin de rendre ce nouveau traitement accessible à tous, il n'en demeure pas moins que son approche cache mal les limites de sa politique. Ces limites sont résumées en trois points par Orsi et Zimmermann :

(1) "Limite d'un modèle fondé sur des injonctions portées par les prix et les subventions au secteur public pour répondre aux questions de solvabilité des pays "; (2) "Limite d'un modèle centré sur le secteur public de santé et qui peine à réduire les problèmes d'accessibilité aux soins et de contrôle des marchés privés ; (3) "Limite enfin d'un modèle qui a centré la production de CTA sur les grands producteurs internationaux et n'a pas su intégrer les capacités endogènes de production des pays » (Orsi et Zimmermann, $2014: 2$ ). 
Les décideurs mondiaux sont ainsi interpellés en cette année 2015 qui marque l'arrivée à échéance des OMD et le passage aux Objectifs de développement durable (ODD) de 2016 à 2030.

\section{Bibliographie}

BAILEY F.G., 1969, Strategems and Spoils. A social anthropology of politics, Oxford, Basil Blackwell.

BASCO L.K., 2004, « Molecular epidemiology of malaria in Cameroon. XIX. Quality of antimalarial drugs used for self-medication ", American Journal of Tropical Medicine and Hygiene, $70: 245-250$.

BENOIST J., 1996, Soigner au pluriel, Essais sur le pluralisme médical, Paris, Karthala.

BRASSEUR P., DRUILHE P., KOUAMOUO J., BRANDICOURT O., DANIS M., MOYOU S.R., 1986, " High level of sensitivity of chloroquine of 72 Plasmodium falciparum isolates from southern Cameroon in January 1985 », American Journal of Tropical Medicine and Hygiene, 35: 711-716.

FASSIN D., 1985, “ Du clandestin à l'officine, les réseaux de vente illicite des médicaments au Sénégal », Cahiers d’Études Africaines, 98 : 161-177.

FASSIN D., 1986, " La vente illicite de médicaments au Sénégal ", Politique Africaine, $23: 123-130$.

FAYE S., LALOU R. et ADJAMAGBO A., 2004, "Soigner les enfants exclusivement à domicile en cas de paludisme en milieu rural sénégalais : un effet de la pauvreté ? ", African Population Studies, Supplement A, 19 / Etude de la population africaine, Supplément A, 19 : 221-240.

FAYE S., 2012, "Responsabiliser les relais communautaires pour le traitement préventif intermittent saisonnier du paludisme (TPI) : enjeux, modalités, défis ", Autrepart, $60: 129-146$.

FOSSO A.L., 2013, " Jeux d'acteurs et d'intérêt autour de l'introduction des ACT au Cameroun ", Revue gabonaise d'anthropologie, 1, I'Harmattan : 135-180.

GRUENAIS M.-E., 2001, « L'État à la conquête de son territoire national. L'exemple de la réforme du système de santé dans la province de l'Extrême Nord du Cameroun ", in GRUENAIS M.-E. (éd.), "Un système de santé en mutation : le cas du Cameroun ", Le bulletin de I'APAD, 21 : 23-47. 
GRUENAIS M.-E., 2004, « Les qualités de l'offre de soins confessionnelle en Afrique subsaharienne ", Autrepart, $26: 29-46$.

JAFFRE Y., 1999. "Farmacie cittadine, farmacie "per terra" ", Africa $e$ mediterraneo, 1 (99): 31-36, traduit en français sous le titre, "Pharmacies des villes, pharmacies "par terre" ", Le bulletin de I'APAD, 17.

LADO L. et FOSSO A.L., 2010, " Récits de maladie et typologie du pluralisme ", in LADO L. (dir.), Le pluralisme médical en Afrique, Yaoundé et Paris, PUCAC et Karthala : 335-358.

LE MARCIS F., FAYE S., SAMB F.B., BADJI M. et LEVEQUE C., 2013, " Les points aveugles de la lutte contre le paludisme en Casamance ", Face à face [en ligne], 12/2013, mis en ligne le 21 octobre 2013, consulté le 20 octobre 2014, faceaface.revues.org/805

MILLS A., 2002, " La science et la technologie en tant que biens publics mondiaux : s'attaquer aux maladies prioritaires des pays pauvres ", Revue d'économie du développement, vol.16/1-2 : 117-139.

MSF, 2003, ACT Now, www.msf.fr/sites/www.msf.fr/files/2003-04-28-MSF1.pdf

OLIVIER DE SARDAN J.P., 2003a, "L'enquête socio-anthropologique de terrain : synthèse méthodologique et recommandations à usage des étudiants ", Etudes et Travaux, 13, Lasdel-Niamey.

OLIVIER DE SARDAN J.P., 2003b, " Pourquoi le malade anonyme est-il si "mal soigné" ? Culture bureaucratique commune et culture professionnelle de la santé ", in JAFFRE Y. et OLIVIER DE SARDAN J.P. (éds.), Une médecine inhospitalière, Paris, Karthala : 265-294.

OLIVIER DE SARDAN J.P., 2008, La rigueur du qualitatif. Les contraintes empiriques de l'interprétation socio anthropologique, Louvain-la-Neuve, Bruylant academia.

ORSI F. et ZIMMERMANN J.-B., 2014, «Le Marché des Antipaludéens: Entre Régulation et Défaillance ", HAL-SHS, https://halshs.archivesouvertes.fr/halshs-01091331.

SAMUELSON P.A., 1954, "The pure theory of public expenditure ", Review of Economics and Statistics, 11: 387-389.

TAVERNE B. et EGROT M., 2015, «Enjeux globaux et locaux d'un "trafic" de médicaments antirétroviraux entre le Sénégal et l'Europe ", in DESCLAUX 
A. et EGROT M. (éds.), Anthropologie du médicament au Sud, Paris, I'Harmattan : 113-128.

TOURE L. et SANOGO Y., 2014, "Les exemptions de paiement du traitement du paludisme pour les enfants de moins de 5 ans et les femmes enceintes: une politique qui dérange ", in OLIVIER DE SARDAN J.P. et RIDDE V. (éds.), Une Politique publique de santé et ses contradictions, Paris, Karthala: 211-232.

VAN DER GEEST S., 1987, "Self-Care and the Informal Sale of Drugs in South Cameroon ", Social Science and Medicine, 25/3 : 293-305.

VIDAL L., 1999, "La transmission, le sida et ses savoirs », L'homme, $150:$ 59-84.

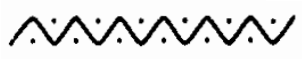

Albert Legrand Fosso est post-doctorant en anthropologie SESSTIM de Marseille

(Sciences économiques et sociales de la santé et traitement de l'information médicale)

E-mail : alfossolegrand@yahoo.fr 